KARRIERE

\title{
Ein Leben für die Organisation
}

\author{
Michael Meyer, Anahid Aghamanoukjan, \\ Johannes Leitner
}

Eine österreichische Studie hat die Karrieren von Führungskräften in Non-Profit-Organisationen mit solchen aus dem gewinnorientierten Sektor verglichen. Ergebnis: Führungskräfte von Non-Profit-Organisationen haben eine unglaublich ausgeprägte Identifikation mit ihrem Unternehmen.

Ist der Non-Profit-Sektor ein attraktives Karrierefeld? Kann man in NonProfit-Organisationen Karriere machen? Viele Menschen werden diese Frage spontan mit Nein oder zumindest mit einem skeptischen »eher nicht « beantworten. Der Mangel an finanziellen Ressourcen wird dabei genauso häufig ins Treffen geführt wie der Mangel an Aufstiegschancen. Hinterfragt man diese Ersteinschätzungen aber, zeigt sich ein anderes Bild. Es gilt mit zwei Missverständnissen aufzuräumen:

\section{- Missverständnis 1: Der Non- Profit-Sektor ist ökonomisch und als Arbeitsmarkt irrelevant.}

Das Gegenteil ist wahr, der Dritte Sektor ein ökonomisch höchst relevanter Arbeitsmarkt. Sieben Prozent der gesamten wirtschaftlich aktiven Bevölkerung sind im Durchschnitt der alten 15 EU-Länder in zivilgesellschaftlichen Organisationen bezahlt beschäftigt (Salamon et al. 2004). Nimmt man dazu die Freiwilligenarbeit, so ergibt sich beispielsweise für Österreich ein Anteil von über 20 Prozent an Arbeit, der in und für Non-Profit-Organisationen geleistet wird. Die regionalen Schwankungsbreiten sind groß: In den Niederlanden sind mehr als 14 Prozent der ökonomisch Aktiven in Non-Profit-
Organisationen beschäftigt, gleiches gilt für große Städte in anderen Ländern. Je höher die ökonomische und demokratische Entwicklung und je stärker urbanisiert ein Land ist, desto wichtiger werden Non-Profit-Organisationen als Karriereoption. Jedenfalls gibt es wenige Branchen, die als Arbeitsmarkt wichtiger sind als der Dritte Sektor. Darüber hinaus sind NonProfit-Organisationen für viele Berufsgruppen - beispielsweise für Angehörige der Pflegeberufe, für Sozialarbeiterinnen und Sozialarbeiter, Behindertenbetreuerinnen und Behindertenbetreuer, für Sporttrainerinnen und Sportrainer - die wichtigste oder einzige Arbeitsmöglichkeit. Insgesamt dürfen Non-Profit-Organisationen als Karrierefeld also keineswegs vernachlässigt werden.

\section{- Missverständnis 2: Karrieren machen einige wenige, der große} Rest arbeitet.

Karriere im wissenschaftlichen Sinn bedeutet aber keineswegs nur den Aufstieg in einsame Höhen, sondern jede Art von Wechsel sozialer Positionen. Insofern sprechen wir ja auch von Drogenkarrieren, Obdachlosenkarrieren etc. Zweifelsohne interessiert aber in Bezug auf Karrieren besonders die Frage nach den Erfolgsfaktoren. Was macht erfolgreich und was bedeutet Karriereerfolg überhaupt?

Im Rahmen des Vienna Career Panel Project (ViCaPP, Internet http://www.vicapp.at) haben wir in einer qualitativen Studie die Karrieren von Führungskräften in Non-Profit-Organisationen $(n=20)$ mit vergleichbaren Karrieren aus dem gewinnorientierten Sektor $(n=40)$ verglichen. Diese Analyse erfolgt vor

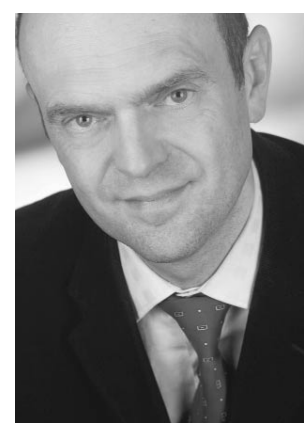

Univ. Prof. Dr. Michael Meyer leitet die Abteilung für NonProfit-Management an der Wirt-

schaftsuniversität Wien, er ist auch wissenschaftlicher Leiter des Professional MBA-Lehrganges für Sozialmanagement (ISMOS) der WU Wien. Arbeitsschwerpunkte: Managerialismus in Non-Profit- Organisationen, Partizipation in der Zivilgesellschaft, Karrieren, Funktionen von Non-Profit- Organisationen.

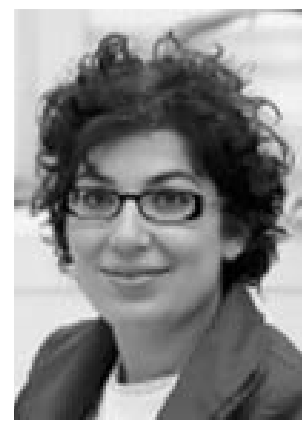

Mag.a Anahid Aghamanoukjan ist wissenschaftliche Mitarbeiterin und Doktorandin an der Abteilung

für Non-Profit Management. Ihre Forschungsschwerpunkte sind Innovationen und Karrieren im Non-Profit-Sektor und Methoden der qualitativen Sozialforschung.

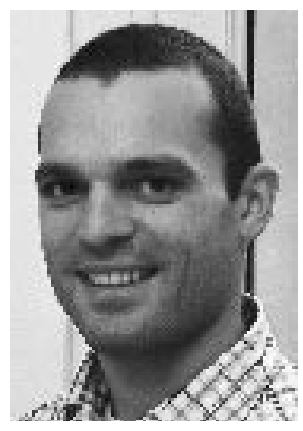

Mag. Johannes Leitner ist wissenschaftlicher Mitarbeiter und Doktorand an Abteilung für Non-

Profit Management der $W U$ Wien. Er forscht zu den Bereichen Karrieren im Non-ProfitSektor, Organizational Slack in Non-Profit-Organisationens, Innovationen und Funktionen des Non-Profit-Sektors. Internet http://www.wuwien.ac.at/npo 

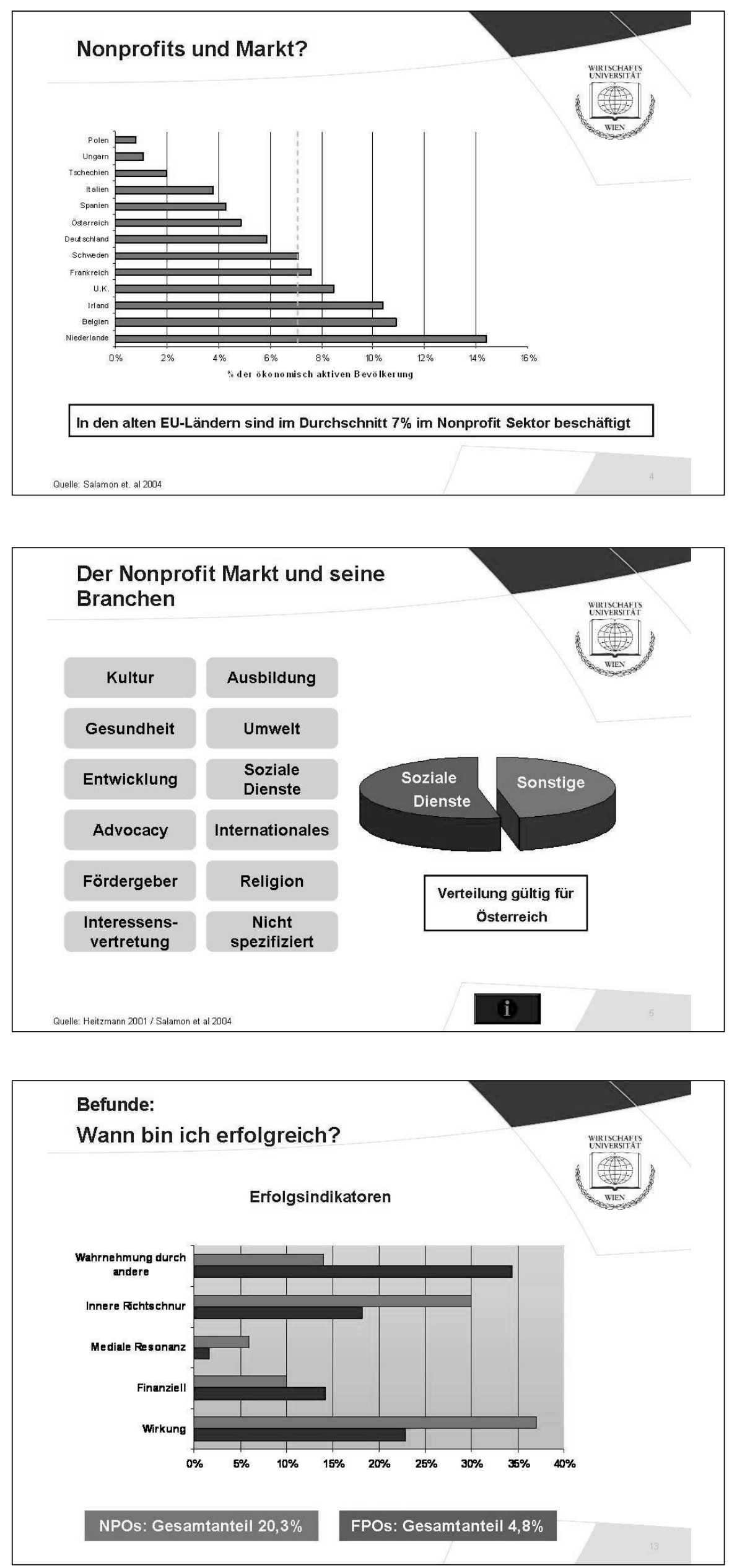

dem Hintergrund zweier dramatischer Veränderungen in den Berufskarrieren insbesondere von Wirtschaftsakademikern, die in dem Forschungsprojekt identifiziert wurden (vgl. Mayrhofer et al. 2005):

(1) Berufslaufbahnen dynamisieren sich rasant. Während Angehörige der 1970er-Kohorte, also Hochschulabsolventen dieses Jahres, während dreißig Jahren durchschnittlich viermal den Job wechselten, tun dies Angehörige der 1990er-Kohorten in nur zehn Jahren ebenso oft.

(2) Subjektiver Karriereerfolg, also Zufriedenheit mit dem beruflichen Werdegang, und objektiver Karriereerfolg (Einkommen, hierarchische Position) klaffen bei vielen deutlich auseinander: Nur 17 Prozent sind objektiv erfolgreich und zufrieden, 20 Prozent sind objektiv erfolgreich und damit unzufrieden (mehr Männer als Frauen), 33 Prozent sind objektiv erfolglos, aber zufrieden (mehr Frauen als Männer), und weitere 30 Prozent sind objektiv und subjektiv erfolglos.

Allein diese beiden Befunde haben starke Bezüge zum Karrierefeld NonProfit-Sektor: Dieser war und ist nämlich vielfach Versuchslabor für die Prekarisierung und Flexibilisierung von Beschäftigungsverhältnissen. Teilzeitarbeit, befristete Verträge, Werkverträge und Scheinselbstständigkeit waren nämlich im Dritten Sektor längst Usus, bevor sie auch von Unternehmen entdeckt wurden. Das Auseinanderklaffen von objektivem und subjektivem Karriereerfolg erklärt schließlich, dass das Karrierefeld NonProfit-Organisationen zunehmend für Menschen attraktiv wird, die über einen wirtschaftlichen Ausbildungshintergrund verfügen. Geld und hierarchischer Aufstieg allein ist für viele kein hinreichender Karrieremotivator.

Vor diesem Hintergrund vergleichen wir Karrieren in Non-Profit-Organisationen und gewinnorientierten Unternehmen hinsichtlich zweier Fragen:

(1) Was wird als Basis für eine erfolgreiche Karriere gesehen? Auf welche Kapitalien - Sozialkapital, ökonomisches Kapital, Kulturkapital (Bourdieu 1983) - werden diese zurückgeführt?

(2) Was ist der Maßstab für Karriereerfolg? Woran ist für die Befragten zur Bemessung ihres subjektiven Karriereerfolges entscheidend? 


\begin{tabular}{|l|l|l|}
\hline & Non-Profit-Organisation & Unternehmen \\
\hline Ökonomisches Kapital & $31 \%$ & $3 \%$ \\
\hline Kulturelles Kapital & $34 \%$ & $32 \%$ \\
\hline Soziales Kapital & $21 \%$ & $41 \%$ \\
\hline Gesamtanteil & $15,5 \%$ & $14 \%$ \\
\hline
\end{tabular}

Tabelle: Relative Häufigkeiten codierter Stellen

\section{Was man für eine Karriere braucht}

Das Fundament für erfolgreiche Karrieren kann anhand von drei Kapitalien skizziert werden, dem Sozialkapital, dem ökonomischen Kapital und dem Kulturkapital:

- Soziales Kapital umschreibt persönliche Netzwerke, Freundschaften und Kontakte.

- Ökonomisches Kapital bezieht sich auf materiellen Reichtum.

- Kulturelles Kapital umschreibt Wissen, Verhaltensweisen, Diplome, Abschlüsse oder Titel, die im Laufe des Lebens erworben wurden.

Unsere Analysen zeigen deutliche Unterschiede bezüglich der Wichtigkeit der verschiedenen Kapitalien als Basis für erfolgreiche Karrieren in Non-Profit-Organisationen und in gewinnorientierten Unternehmen. Alleine eine erste quantitative Auswertung der relevanten Textpassagen zeigt interessante Muster auf (vgl. Tabelle):

- Ökonomisches Kapital wird von Non-Profit-Führungskräften weitaus häufiger erwähnt, was auf eine höhere Bedeutung in diesem Sektor hinweist. Dabei wird nahezu immer der Mangel an finanziellen Mitteln beklagt, ein Thema, das gewinnorientierten Managerinnen und Managern kein Kopfzerbrechen bereitet. Ein wichtiges Detail in diesem $\mathrm{Zu}$ sammenhang ist, dass ökonomisches Kapital für Manager von NonProfit- Organisationen nicht nur in Form von persönlichem Eigentum und Einkommen Bedeutung hat, sondern auch als organisationales Einkommen. Die Grenzen zwischen persönlichem und organisationalem ökonomischen Kapital ver- schwimmen sehr oft, was durch eine unglaublich ausgeprägte Identifikation der Manager von NonProfit-Organisationen mit ihrer Organisation zu erklären ist. Somit verwundert es nicht, wenn die Akquisition finanzieller Mittel für die Organisation als persönlicher Karriereschritt interpretiert wird, selbst wenn damit keine Gehaltserhöhungen einhergehen.

- Kulturelles Kapital scheint in beiden Karrierefeldern etwa gleich viel Gewicht zu haben. Die Differenzen schäfts- und Kundenkontakte nennen. Führungskräfte in Non-ProfitOrganisationen hingegen zeigen, dass für sie das Bauen von Brücken Priorität hat. Beziehungen im NonProfit-Sektor sind wichtig, über Organisationsgrenzen hinweg; im Gegensatz zu Kontakten in der eigenen Organisation. Auffällig ist auch, dass das Beziehungsgeflecht der Manager von Non-Profit-Organisationen durch Entgrenzung (»blurring boundaries«) zwischen privaten und beruflichen Kontakten gekennzeichnet ist.

\section{Wie man Erfolg hat}

Im Zuge der qualitativen Analyse des Datenmaterials wurden fünf Kriterien gefunden, die als Indikatoren zur Erfolgsmessung für Manager in Non-Profit-Organisationen dienen. Diese sind die Wirkung des eigenen Handelns, das Geld, die mediale Resonanz sowie der gute Ruf und das Image der Organisation:

\section{"Non-Profit-Organisationen werden sich niemals mit einer reinen Dienstleister-Rolle zufrieden geben, sondern immer auch Meinungsbildung ausüben wollen "}

werden bei einer detaillierten Betrachtung evident. So sind die Karrierewege im Non-Profit- Bereich, der diffuse Anforderungen an seine Akteure stellt und nach Universalkompetenzen verlangt, durch eine Abfolge komplementärer Ausbildungen gekennzeichnet. Ausgebildete Sozialarbeiter qualifizieren sich in Richtung Management, während Wirtschaftsakademiker eher psychosoziale Weiterbildungen besuchen. Die Motivation für Weiterbildung ist im Non-Profit Sektor hochgradig intrinsisch begründet, hingegen zeigen Manager aus dem Unternehmenssektor überwiegend extrinsische Motivationsmuster.

- Soziales Kapital wird von Managern in Unternehmen deutlich häufiger erwähnt, wobei diese in erster Linie Kollegen, Vorgesetzte sowie Ge-
- Zentrale Bedeutung für den Erfolg hat die Wirkung des eigenen Handelns: Etwas Bewegen zählt, sei es für die Klienten, in der Organisation - dies steigt mit Führungsfunktion -, im beruflichen Umfeld (z. B. das Setzen neuer Standards in der Behindertenarbeit) oder mit etwas Abstand in der Gesellschaft. Wirkung ist offenbar von zentraler Bedeutung, wird aber realistisch gesehen. Niemand kann mehr die Gesellschaft verändern.

- Geld spielt im Non-Profit Sektor eine große Rolle. Allerdings nicht so sehr als persönliches Einkommen oder Gewinn der Organisation. Erfolgreich ist derjenige, der viel Geld für die Organisation aufstellen kann. Das eigene Einkommen kommt nur selten vor, und dann nur als Hygienefaktor. 
- Ein prominenter Erfolgsindikator ist hingegen die mediale Resonanz des eigenen Tuns - und das nicht nur bei jenen, die in Advocacy-NonProfit-Organisationen arbeiten, also in Organisationen, die Interessenvertretung, Lobbying oder politische Einflussnahme als ihre Aufgaben definieren. Medienresonanz kann für die eigene Karriere durchaus dienlich sein und ist gleichzeitig ein Indikator für den eigenen Karriereerfolg - auch wenn es dabei natürlich um die gute Sache geht. Das beweist, dass Non-Profit-Organisationen sich niemals mit einer reinen Dienstleisterrolle zufrieden geben werden, sondern immer auch Meinungsbildung ausüben. Öffentliche Wahrnehmung ist in vielen Bereichen von Non-ProfitOrganisationen jedenfalls zentraler Erfolgsfaktor - und hier können Dienst an der Sache und persönliche Eitelkeiten verschwimmen.

- Von allen Kriterien sind der "gute Ruf « und das »Image« die wichtigsten Erfolgsindikatoren für die eigene Organisation und damit für den Karriereerfolg. Auch wenn nicht konkret auf Medienberichte angesprochen wird, ist eine abstrakte Öffentlichkeit wichtigste Bezugsgruppe - die Wahrnehmung des eigenen Wirkens durch andere somit höchst bedeutsam. Damit sind meist die Fachöffentlichkeit oder die Branche gemeint, seltener die eigenen Mitarbeiter oder private Bezugsgruppen wie Familie oder Freunde.

\section{Literatur}

Bourdieu, Pierre (1983): Ökonomisches Kapital, kulturelles Kapital, soziales Kapital. In: Kreckel, R. (Hg.): Soziale Ungleichheit. Soziale Welt, Sonderband 2. 183-198.

Mayrhofer, Wolfgang/Meyer, Michael/Steyrer, Johannes (Hg.). (2005): Macht? Erfolg? Reich? Glücklich? Einflussfaktoren auf Karrieren. Wien.

Salamon, Leister M./Sokolewski, Wojciech/Associates (2004): Global Civil Society. Dimensions of the Nonprofit Sector. Bloomfield.

\section{Öffentliche Beschaffungs- politik im Wandel}

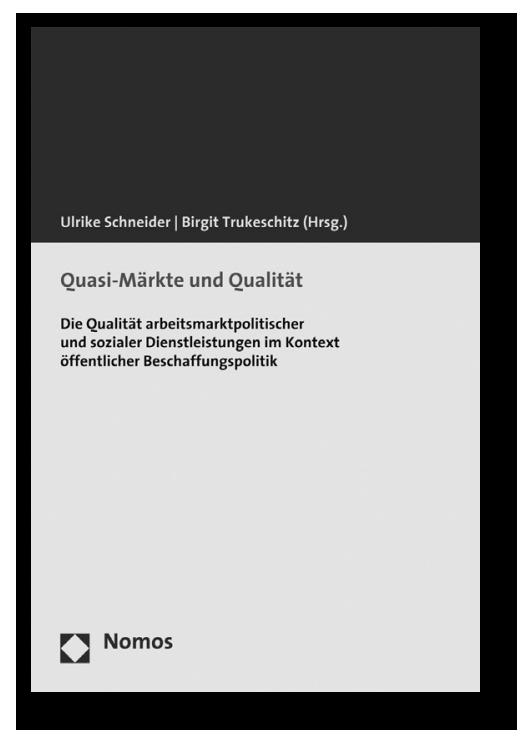

\section{Quasi-Märkte und Qualität}

Die Qualität arbeitsmarktpolitischer und sozialer Dienstleistungen im Kontext öffentlicher Beschaffungspolitik

Herausgegeben von Prof. Dr. Ulrike Schneider, Department Volkswirtschaft, Wirtschaftsuniversität Wien. Leitung des Instituts für Sozialpolitik und des Forschungsinstituts für Altersökonomie und Dr. Birgit Trukeschitz, Institut für Sozialpolitik, Wirtschaftsuniversität Wien

2007, 310 S., brosch., 24,90 €, ISBN 978-3-8329-3073-8

Die öffentliche Beschaffungspolitik im Sozialwesen befindet sich im Wandel. Durch Deregulierung und Öffnung der Märkte, die über die europäische Politik beschleunigt werden, kommen verstärkt marktorientierte Beauftragungen in Bereichen zur Anwendung, die zuvor über Förderungen bzw. auf Basis direkt vergebener Aufträge öffentlich finanziert wurden. Wissenschaftliche Studien zu den Effekten der neuen öffentlichen Beschaffungspolitik im Sozialwesen sind bislang rar. Dort wo eine erste Bilanz vorhanden ist, liegt der Fokus meist auf der Kostenwirkung wettbewerblicher Beschaffungsverfahren.

Dieses Buch geht der Frage nach, wie unterschiedliche Verfahren der öffentlichen Beschaffung arbeitsmarktpolitischer und sozialer Dienstleistungen die Qualität der Angebote von und die Arbeitssituation in Organisationen der Sozialwirtschaft in Österreich beeinflussen.

Die Autorlnnen befassen sich mit dem Konzept der Qualität, diskutieren ausgewählte Beschaffungsverfahren und präsentieren Befunde zu den Handlungsspielräumen und Qualitätswirkungen unterschiedlicher öffentlicher Beschaffungsmodi aus ökonomischer, organisationswissenschaftlicher und rechtswissenschaftlicher Sicht.

Bitte bestellen Sie bei Ihrer Buchhandlung oder bei Nomos | Telefon 07221/2104-37 | Fax -43 | www.nomos.de | sabine.horn@nomos.de 